\title{
ARTICLE
}

Clinical Study

\section{Phase 1 trial of olaparib and oral cyclophosphamide in $B R C A$ breast cancer, recurrent $B R C A$ ovarian cancer, non- $B R C A$ triple-negative breast cancer, and non-BRCA ovarian cancer}

\author{
Chee Khoon Lee ${ }^{1,2}$, Clare Scott $^{3,4,5}$, Geoffrey J. Lindeman ${ }^{3,4,5}$, Anne Hamilton ${ }^{3}$, Elizabeth Lieschke ${ }^{4}$, Emma Gibbs ${ }^{2}$, Rebecca Asher $^{2}$,
} Heath Badger ${ }^{6}$, Robin Paterson ${ }^{6}$, Lauren Macnab ${ }^{6}$, Edmond Michael Kwan ${ }^{3}$, Prudence A. Francis ${ }^{3}$, Frances Boyle ${ }^{6,7}$ and Michael Friedlander ${ }^{8}$

BACKGROUND: We conducted a Phase 1 study to evaluate safety and activity of olaparib tablets and oral cyclophosphamide. METHODS: Patients had metastatic breast cancer (BC) or recurrent high-grade serous ovarian cancer (HGSOC), performance status $0-2$, and $\leq 3$ lines of prior therapy. Patients were treated using a dose escalation strategy with cohort expansion once maximal tolerated dose (MTD) was determined. Dose level 1 (DL1): olaparib $300 \mathrm{mg}$ bid, cyclophosphamide $50 \mathrm{mg}$ on days 1, 3, and 5, weekly. DL2: olaparib $300 \mathrm{mg}$ bid, cyclophosphamide $50 \mathrm{mg}$, days 1-5 weekly.

RESULTS: Of 32 patients, 23 had HGSOC (germline BRCA mutation [gBRCAm] 70\%) and 9 had BC (gBRCAm 67\%). Four were treated at DL1 and 28 at DL2, the MTD. Haematological adverse events (AEs) were most common: grade 3/4 AEs: lymphopenia 75\%, anaemia $31 \%$, neutropenia $37 \%$, thrombocytopenia $47 \%$. Two permanently discontinued treatment due to haematological AEs. In $\mathrm{BC}$, no objective response was reported. Unconfirmed objective response was $48 \%$ and $64 \%$ for all HGSOC and gBRCAm subset, respectively. CA125 responses were $70 \%$ (all HGSOC) and $92 \%$ (gBRCAm).

CONCLUSIONS: In HGSOC and BC, olaparib $300 \mathrm{mg}$ bid and cyclophosphamide $50 \mathrm{mg}$ on days 1-5 weekly were tolerable and active, particularly in $\mathrm{g} B R C A \mathrm{~m}$, and is worthy of further investigation.

British Journal of Cancer (2019) 120:279-285; https://doi.org/10.1038/s41416-018-0349-6

\section{INTRODUCTION}

Olaparib is a first-in-class potent oral poly (ADP-ribose) polymerase (PARP) inhibitor. Monotherapy studies have demonstrated acceptable toxicity and activity in metastatic breast cancer ${ }^{1-5}$, and recurrent ovarian cancer ${ }^{1-4,6,7}$ in patients with germline $B R C A$ mutations (gBRCAm). Low-grade nausea, vomiting, fatigue, and anaemia were the most frequently reported adverse events (AEs). Response rates varied from 0 to $60 \%$ and 26 to $41 \%$ for gBRCAm breast and ovarian cancer patient cohorts, respectively.

Inhibition of base-excision DNA repair by olaparib potentiates the DNA damage and cytotoxicity caused by platinum chemotherapy, leading to an increase in genomic instability and tumour cell death. ${ }^{8,9}$ Indeed, studies of the combination of olaparib and platinum chemotherapy followed by maintenance olaparib have reported high response rates and prolonged progression-free survival (PFS) ${ }^{10}$ for $\mathrm{gBRCAm}$ recurrent ovarian carcinomas but with a significant increase in myelotoxicity ${ }^{10-12}$ requiring a reduction of the doses of both carboplatin and olaparib for safe administration, such that the combination is not recommended. Similar findings were reported in metastatic triple-negative breast cancer, where combination of olaparib with paclitaxel resulted in high rates of neutropenia despite secondary prophylaxis with granulocytecolony stimulating factor. ${ }^{13}$

Cyclophosphamide is a bifunctional alkylating agent with modest monotherapy activity in advanced breast ${ }^{14,15}$ and ovarian cancers. ${ }^{16}$ Cyclophosphamide damages DNA through the formation of complex inter-strand crosslinks, which could potentiate the activity of PARP inhibitors and lead to greater cytoxicity. ${ }^{17}$ Preclinical studies also provide some evidence to support combination therapy with alkylating chemotherapy and PARP inhibitors, particularly those that trap PARP-DNA complexes effectively. ${ }^{18,19}$ Metronomic cyclophosphamide is administered orally at a low dose on a continuous schedule for prolonged periods and is well tolerated with low rates of myelosuppression. Therefore, theoretically, metronomic cyclophosphamide could be combined with a PARP inhibitor without associated significant toxicity and with increased cytotoxicity.

However, a randomised Phase 2 trial of combination veliparib and metronomic oral cyclophosphamide in heavily pre-treated ovarian cancer patients failed to demonstrate improvement in either the response rate or PFS over cyclophosphamide monotherapy. ${ }^{20}$ Veliparib is a less efficient trapper of PARP than

\footnotetext{
${ }^{1}$ St George Hospital, Sydney, NSW, Australia; ${ }^{2}$ National Health and Medical Research Council (NHMRC) Clinical Trials Centre, Sydney, NSW, Australia; ${ }^{3}$ Peter MacCallum Cancer Centre, Melbourne, VIC, Australia; ${ }^{4}$ Walter and Eliza Hall Institute of Medical Research, Melbourne, VIC, Australia; ${ }^{5}$ The Royal Melbourne Hospital, Melbourne, VIC, Australia; ${ }^{6}$ Breast Cancer Trials Australia \& New Zealand, Newcastle, NSW, Australia; ${ }^{7}$ The Mater Hospital, North Sydney, NSW, Australia and ${ }^{8}$ Prince of Wales Hospital, Sydney and Prince of Wales Clinical School, University of New South Wales, Sydney, NSW, Australia Correspondence: Chee Khoon Lee (chee.lee@ctc.usyd.edu.au)
} 
olaparib, $^{18}$ however, and this trial used a potentially subtherapeutic dose of veliparib $60 \mathrm{mg}$ daily, compared to the recommended monotherapy dose of $300-400 \mathrm{mg}$ twice daily, in order to enable continuous cyclophosphamide $50 \mathrm{mg}$ daily dosing.

We conducted the SOLACE trial, a Phase 1 study of olaparib tablets and metronomic oral cyclophosphamide in pre-treated patients with metastatic $\mathrm{g} B R C A \mathrm{~m}$ breast cancer, recurrent $\mathrm{g} B R C A \mathrm{~m}$ high-grade serous ovarian cancer (HGSOC), non-BRCA triplenegative breast cancer, and non-BRCA HGSOC. The objectives of the SOLACE trial were to establish the safety, tolerability, maximum tolerated dose (MTD), and preliminary efficacy of the combination. Unlike the previous veliparib-cyclophosphamide study, our trial adopted the strategy of maintaining the recommended monotherapy dose of olaparib tablets while escalating the metronomic administration of oral cyclophosphamide, using a dose-escalation design.

\section{PATIENTS AND METHODS}

The SOLACE trial (ANZCTRN: 12613000924752) was an investigator-initiated study sponsored by Breast Cancer Trials Australia and New Zealand. Astrazeneca provided olaparib for this study. Ethical approval for the conduct of the study was provided by the appropriate Human Research Ethics Committees at each of the three participating clinical sites. All patients provided written informed consent.

\section{Eligibility criteria}

Eligible patients with recurrent or metastatic disease after standard therapies had measurable (Response Evaluation Criteria in Solid Tumors [RECIST] v1.1) or non-measurable HGSOC with an elevated CA125 evaluable using Gynecologic Cancer InterGroup (GCIG) CA125 response criteria, ${ }^{21}$ an Eastern Cooperative Organization Group performance status of $\leq 2$, and adequate bone marrow, liver, and kidney function. Documentation of $\mathrm{gBRCAm}$ status was not required. Prior exposure to a PARP inhibitor was not allowed. Patients who were unable to swallow pills or those with uncontrolled intercurrent illness, including brain metastases or gastrointestinal conditions that might predispose to drug intolerance or poor drug absorption were also excluded.

\section{Study design}

The trial design used a backbone of olaparib tablets $300 \mathrm{mg}$ twice daily continuously for all patients starting on day 1 of a 3-weekly cycle. Oral cyclophosphamide $50 \mathrm{mg}$ was given, starting on day 1 of a 3-weekly cycle, on an increasing number of days each week (dose level (DL) 1 for 3 days, DL2 for 5 days, and DL3 for 7 days) to sequential cohorts of patients (Table 1). Dose levels with lower olaparib doses were included in the design in the event of unexpected toxicity, but these were not activated. Eligible patients were enrolled in a ' $3+3$ ' dose escalation format, with cohort expansion at the MTD.
Patients were evaluated for toxicity and response over 24 weeks or 8 cycles of treatment, with each treatment cycle administered over 3 weeks. Toxicity was graded using the Common Terminology Criteria for Adverse Events v4.0, and tumour response was assessed at weeks 6,15 , and 24; response and progression was evaluated using RECIST v1.1 for patients with measurable disease and CA125 (for the HGSOC cohort) as defined by GCIG criteria. ${ }^{21}$ Study treatment was discontinued for symptomatic disease progression, intercurrent illness, unacceptable toxicity, or patient withdrawal of consent. In the absence of disease progression, patients were allowed to continue with one or both study agents beyond 24 weeks at the discretion of the study physicians. Patients were also allowed to continue study treatment in the presence of radiological or CA125 progression for asymptomatic or minimally symptomatic patients at the discretion of the study physicians.

Definitions of dose-limiting toxicity and maximum tolerated dose The primary endpoint of this study was to determine the recommended Phase 2 dose (RP2D) of the olaparib and cyclophosphamide combination, defined by the MTD or the highest protocol-defined dose in the absence of dose-limiting toxicity (DLT). DLT was any of the following events that occurred during the first 6 weeks of therapy: neutrophil count $<0.5 \times 10^{9} / \mathrm{L}$ without fever and lasting for $>5$ days, neutropenic sepsis, platelet counts $<$ $25 \times 10^{9} / \mathrm{L}$, any grade 3 or 4 non-haematological adverse event (AE) despite appropriate supportive measures, any $A E$ not otherwise described that resulted in a treatment delay of $>21$ consecutive days and repeated requirement for blood transfusions within the first 2 cycles ( 6 weeks). In the determination of the MTD, recurrent AEs that were encountered beyond the first 6 weeks of therapy were also taken into consideration (Supplementary Table 1).

Pharmacokinetic studies

For the DL1 cohort, plasma samples to quantify serum olaparib level were collected $1,2,6$, and $12 \mathrm{~h}$ after ingestion of the morning dose of olaparib on day 7 (following 1 week of olaparib monotherapy). Plasma samples were collected at the same time points on day 14 (after patients had received 1 week of combination therapy with olaparib and cyclophosphamide). For the DL2 cohort, samples were collected at the same time points on days 8 of cycles 1 and 2 for patients receiving combination olaparib-cyclophosphamide. Blood samples were centrifuged and stored until measurement using validated assays with a lower limit of quantitation of $0.02 \mu \mathrm{g} / \mathrm{mL}$ for olaparib. ${ }^{22}$

Statistical analyses

Baseline demographics were summarised using the median number and range for continuous variables, and frequency and percentages for categorical variables. All patients treated with at least one dose of the study drugs were evaluated for efficacy and toxicity. For efficacy analyses, objective response rates (ORR) and stable disease for at least 6 weeks (SD6) were computed

\begin{tabular}{|llll|}
\hline Table 1. & Patient cohort at different dose levels & & \\
\hline $\mathrm{DL}$ & Olaparib tablet & Cyclophosphamide tablet & Number of patients \\
\hline-2 & $200 \mathrm{mg}$ bid & $50 \mathrm{mg}$, days 1, 3 and 5 every week & 0 \\
-1 & $250 \mathrm{mg}$ bid & $50 \mathrm{mg}$, days 1, 3 and 5 every week & 0 \\
1 & $300 \mathrm{mg}$ bid & $50 \mathrm{mg}$, days 1, 3 and 5 every week & 4 \\
2 & $300 \mathrm{mg}$ bid & $50 \mathrm{mg}$, days 1-5 every week & $28^{\mathrm{a}}$ \\
3 & $300 \mathrm{mg}$ bid & $50 \mathrm{mg}$, days 1-7 every week & 0 \\
\hline$D L$ dose level, $m g$ milligram, bid twice per day & & \\
a7 patients were initially enrolled in DL2. When this was determined to be the MTD, another 21 patients were enrolled in DL2 as a separate expansion cohort
\end{tabular}


Phase 1 trial of olaparib and oral cyclophosphamide in BRCA breast... CK Lee et al.

Table 2. Baseline characteristics of the trial participants

\begin{tabular}{|c|c|c|}
\hline Characteristics & $\begin{array}{l}\text { Breast cancerN } \\
\text { (\%) }\end{array}$ & HGSOCN (\%) \\
\hline \multicolumn{3}{|l|}{ Sex } \\
\hline Female & $9(100)$ & $23(100)$ \\
\hline \multicolumn{3}{|l|}{ Age (years) } \\
\hline Median & 46 & 60 \\
\hline Range & $33-64$ & $37-84$ \\
\hline \multicolumn{3}{|l|}{ ECOG performance status } \\
\hline 0 & $6(67)$ & $13(57)$ \\
\hline 1 & $3(33)$ & $10(43)$ \\
\hline \multicolumn{3}{|l|}{$g B R C A$ status } \\
\hline$B R C A$ negative & $3(33)$ & $6(26)$ \\
\hline$g B R C A 1$ & $2(22)$ & $10(44)$ \\
\hline$g B R C A 2$ & $4(45)$ & $6(26)$ \\
\hline Unknown & 0 & $1(4)$ \\
\hline \multicolumn{3}{|l|}{ Breast Cancer cohort } \\
\hline ER positive & 6 & \\
\hline PR positive & 4 & \\
\hline ER, PR, and HER2 negative & 3 & \\
\hline \multicolumn{3}{|l|}{ Lines of prior therapy } \\
\hline 1 & $2(22)$ & 9 (39) \\
\hline 2 & $4(45)$ & $8(35)$ \\
\hline 3 & $3(33)$ & $6(26)$ \\
\hline \multicolumn{3}{|l|}{ Breast Cancer cohort } \\
\hline Prior anthracycline & $7(78)$ & \\
\hline Prior taxane/anti-tubulin & $8(89)$ & \\
\hline Prior platinum ${ }^{a}$ & $6(67)$ & \\
\hline \multicolumn{3}{|l|}{ Ovarian Cancer cohort } \\
\hline $\begin{array}{l}\text { Lines of prior platinum-based } \\
\text { therapy }(1 / 2 / 3)\end{array}$ & & $\begin{array}{l}9(39) / 10(44) / 4 \\
(17)\end{array}$ \\
\hline Prior bevacizumab & & $6(26)$ \\
\hline $\begin{array}{l}\text { Platinum-sensitive at first } \\
\text { relapse }\end{array}$ & & $22(96)$ \\
\hline $\begin{array}{l}\text { Platinum-sensitive at trial } \\
\text { enrolment }\end{array}$ & & $15(65)$ \\
\hline
\end{tabular}

separately for breast and HGSOC cohorts as well as for the $\mathrm{gBRCAm}$ cohorts regardless of tumour types. ORR was defined as the sum of complete responses and partial responses. DCR was defined as the sum of complete responses, partial responses and stable disease for $\geq 6$ weeks. Response duration was not evaluated due to infrequent nature of tumour assessments (weeks 6, 15, and 24 only). The number of patients with each type of worst grade toxicity experienced was summarised as percentages. PFS duration was defined as the time from study commencement to objective tumour progression or death. The Kaplan-Meier approach was used for analysis of PFS.

\section{RESULTS}

Patient characteristics

Between June 2014 and August 2016, 32 women were enrolled (dose escalation cohort: $N=11$; dose expansion cohort: $N=21$ ), with 16 patients still receiving olaparib monotherapy at the time
Table 3. Treatment-related adverse events by maximum grade per patient

\begin{tabular}{|c|c|c|c|c|c|}
\hline & Grade 1 & Grade 2 & Grade 3 & Grade 4 & Total \\
\hline $\begin{array}{l}\text { Lymphocyte count } \\
\text { decrease }\end{array}$ & $2(6 \%)$ & $6(19 \%)$ & $21(66 \%)$ & $3(9 \%)$ & 32 \\
\hline Nausea/vomiting & $21(66 \%)$ & $8(25 \%)$ & $1(3 \%)$ & & 30 \\
\hline $\begin{array}{l}\text { White blood count } \\
\text { decrease }\end{array}$ & $3(9 \%)$ & $12(38 \%)$ & $14(44 \%)$ & $1(3 \%)$ & 30 \\
\hline Anaemia & $4(13 \%)$ & 14 (44\%) & 10 (31\%) & & 28 \\
\hline Fatigue & 17 (53\%) & 10 (31\%) & & & 27 \\
\hline $\begin{array}{l}\text { Neutrophil count } \\
\text { decrease }\end{array}$ & $2(6 \%)$ & $12(38 \%)$ & $11(34 \%)$ & $1(3 \%)$ & 26 \\
\hline Infection & $3(9 \%)$ & $15(47 \%)$ & $3(9 \%)$ & & 21 \\
\hline $\begin{array}{l}\text { Platelet count } \\
\text { decrease }\end{array}$ & $16(50 \%)$ & $2(6 \%)$ & & & 18 \\
\hline $\begin{array}{l}\text { Abdominal pain, } \\
\text { bloating, dyspepsia, } \\
\text { distension }\end{array}$ & $12(38 \%)$ & $2(6 \%)$ & $1(3 \%)$ & & 15 \\
\hline Non-abdominal pain & $13(41 \%)$ & $2(6 \%)$ & & & 15 \\
\hline Constipation & $9(28 \%)$ & $2(6 \%)$ & $1(3 \%)$ & & 12 \\
\hline Headache & $10(31 \%)$ & $1(3 \%)$ & & & 11 \\
\hline Dizziness & $10(31 \%)$ & & & & 10 \\
\hline Dysgeusia & $10(31 \%)$ & & & & 10 \\
\hline Cough & $7(22 \%)$ & $2(6 \%)$ & & & 9 \\
\hline Diarrhoea & $8(25 \%)$ & & $1(3 \%)$ & & 9 \\
\hline Anorexia & 7 (22\%) & $1(3 \%)$ & & & 8 \\
\hline Dyspnoea & 7 (22\%) & $1(3 \%)$ & & & 8 \\
\hline Oral mucositis & $8(25 \%)$ & & & & 8 \\
\hline Fever & $3(9 \%)$ & & & & 3 \\
\hline Hypertension & $1(3 \%)$ & $1(3 \%)$ & $1(3 \%)$ & & 3 \\
\hline $\begin{array}{l}\text { Decrease in serum } \\
\text { phosphate }\end{array}$ & & $3(9 \%)$ & & & 3 \\
\hline Nail changes & $2(6 \%)$ & & & & 2 \\
\hline Vascular disorders & $1(3 \%)$ & $1(3 \%)$ & & & 2 \\
\hline $\begin{array}{l}\text { Decrease in serum } \\
\text { potassium }\end{array}$ & $1(3 \%)$ & & & & 1 \\
\hline $\begin{array}{l}\text { Decrease in serum } \\
\text { magnesium }\end{array}$ & & $1(3 \%)$ & & & 1 \\
\hline $\begin{array}{l}\text { Decrease in serum } \\
\text { sodium }\end{array}$ & $1(3 \%)$ & & & & 1 \\
\hline $\begin{array}{l}\text { Increase in serum } \\
\text { creatinine }\end{array}$ & & & $1(3 \%)$ & & 1 \\
\hline
\end{tabular}

of data cut-off in September 2017. Table 2 summarises the baseline patient characteristics. HGSOC was the most common tumour type $(N=23[72 \%])$, and the majority of these patients had gBRCAm (overall $N=22$ [69\%], HGSOC $N=16$, breast cancer $N=6$ ). Patients had a good performance status, with a median age of 56 years. A total of $28 \%$ of patients had received three lines of systemic therapy; Supplementary Table 2 summarises in detail the different types of prior therapy.

Dose optimisation and treatment-related adverse events We have identified the RP2D as olaparib tablets $300 \mathrm{mg}$ twice daily, and cyclophosphamide tablets $50 \mathrm{mg}$ on days 1-5 each week. Despite no DLT's being observed with olaparib plus cyclophosphamide during the first 6 weeks of therapy at DLs 1 and 2, patients did not proceed to DL3 due to recurrent haematological AEs observed beyond 6 weeks (Table 1). A total of $16(50 \%)$ patients stopped treatment before or at 24 weeks; only two patients stopped early due to unacceptable toxicity. The 
a

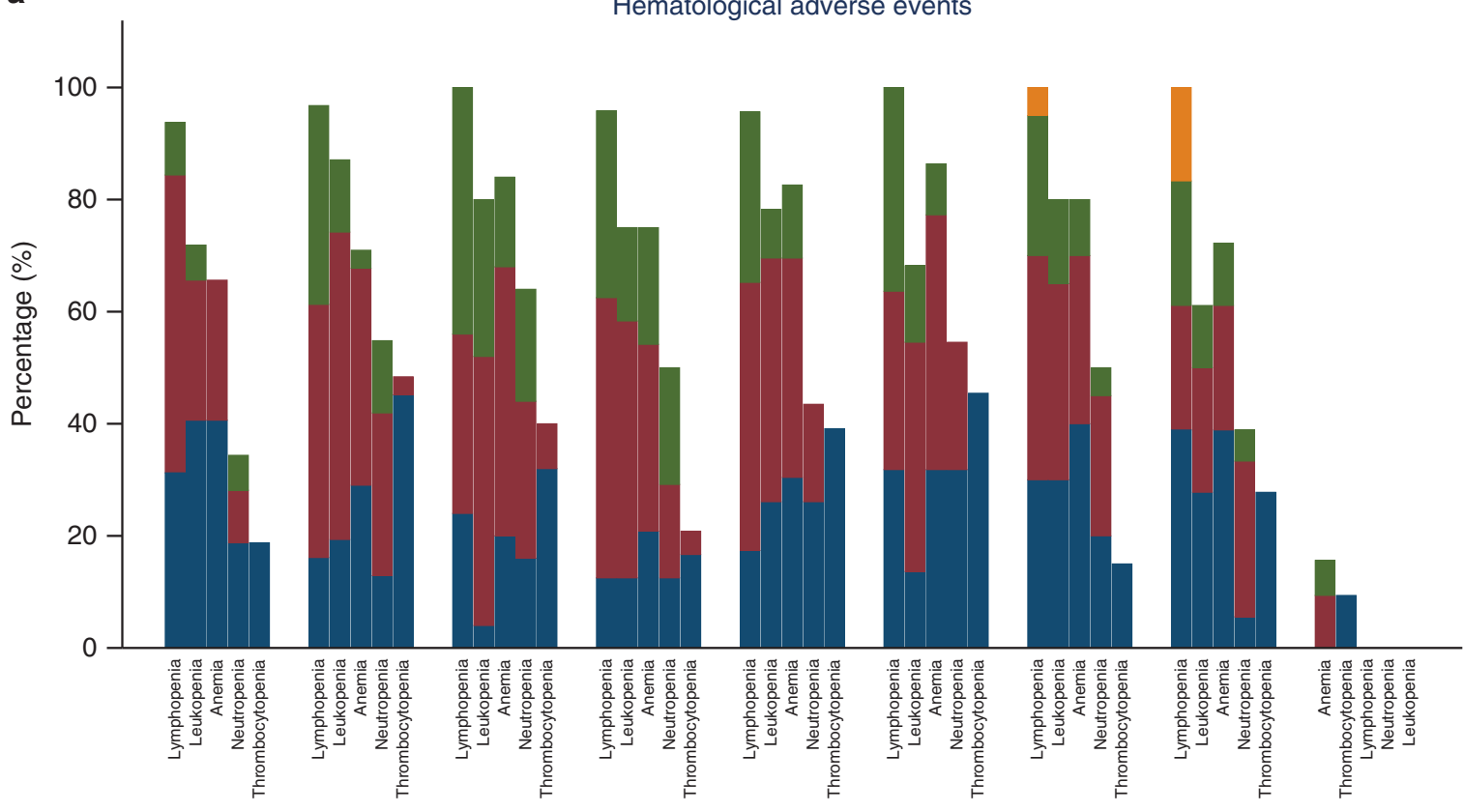

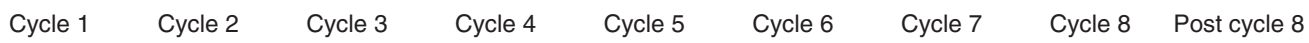

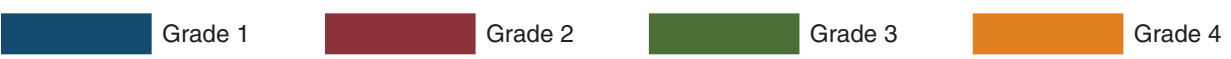

b

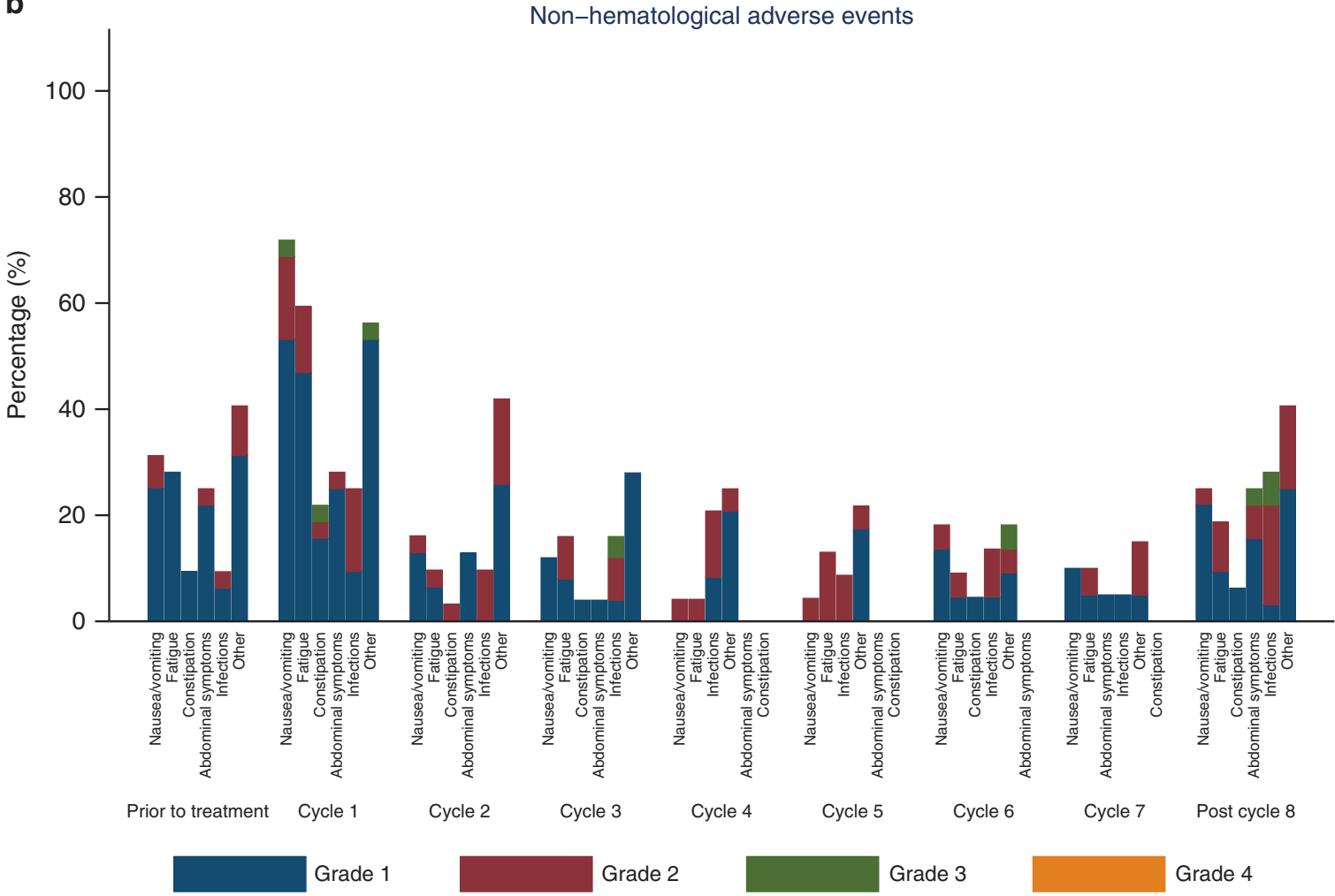

Fig. 1 Frequency of adverse events according to $\mathbf{a}$ haematological and $\mathbf{b}$ non-haematological types experienced after each treatment cycle

remaining 16 patients (50\%) continued with study treatment beyond 24 weeks; all of these patients continued olaparib monotherapy after stopping cyclophosphamide. The median duration of protocol-defined therapy during the eight cycles was 5.5 months (range: 0.7-5.5). Among patients who continue olaparib beyond the eighth cycle, the median duration of therapy was 5.0 months (range: $0.7-23.5+$ ).

Table 3 summarises any-grade AE's in all patients. Grades 3 and $4 \mathrm{AE}^{\prime}$ 's occurred in 84 and $13 \%$ of patients, respectively. Haematological AE's were the most common: lymphopenia (grade 
a

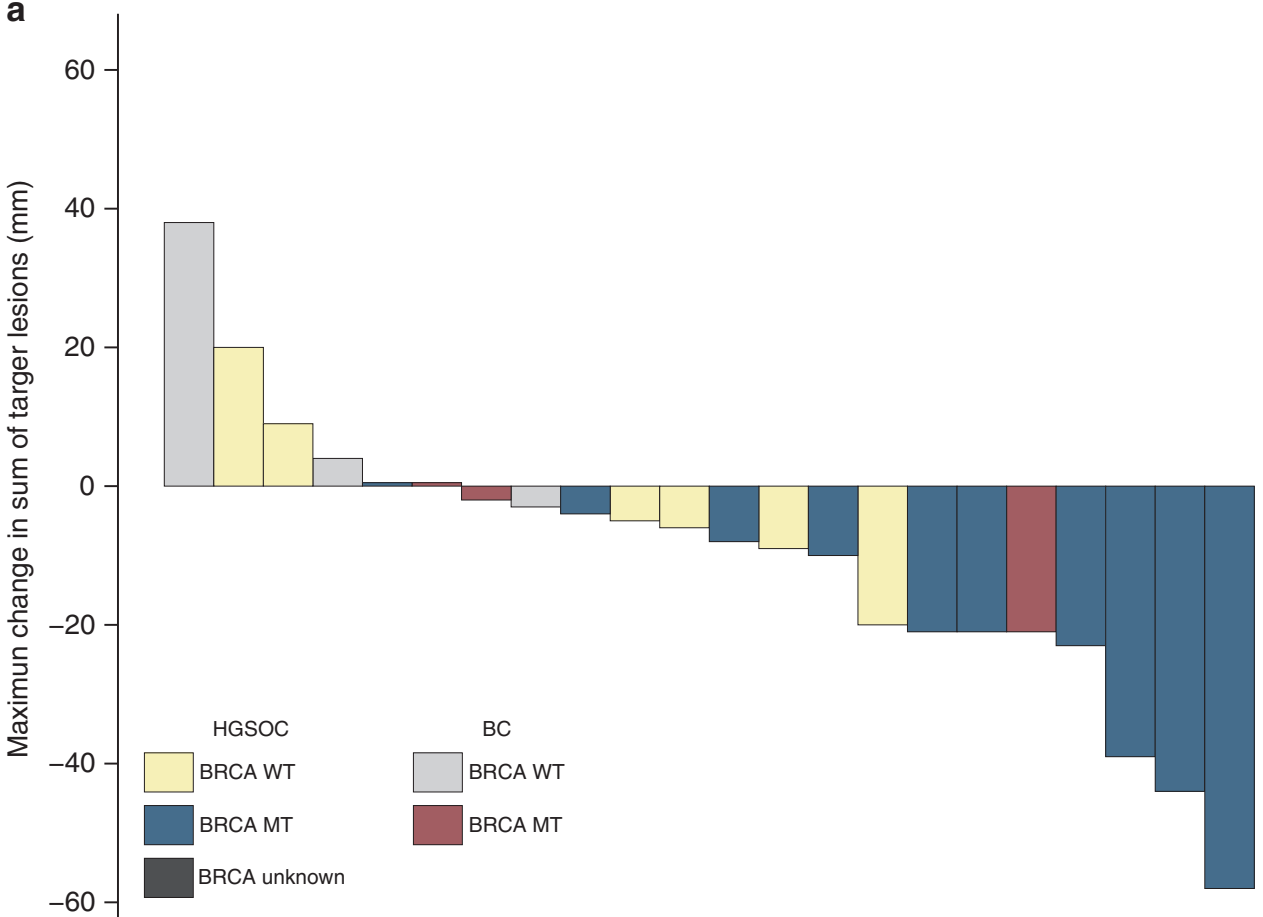

b Patient ID

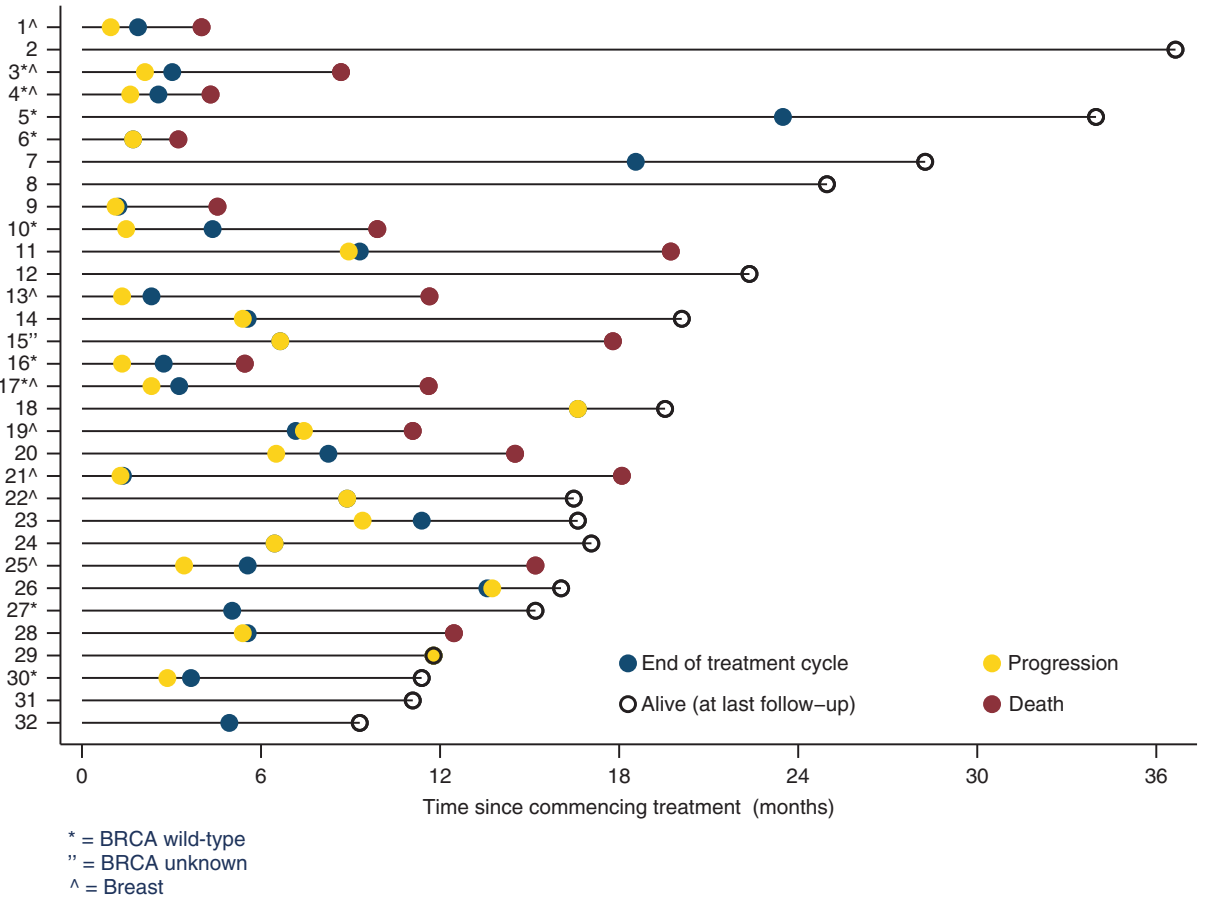

Fig. 2 a Best response for target lesions by patient based on maximal percentage of tumour reduction. b Swimmer plot depicts individual patients as lines. BC metastatic triple-negative breast cancer, HGSOC high-grade serous ovarian cancer

1/2: $25 \%$, grade 3/4: $75 \%$ ), anaemia (grade $1 / 2: 57 \%$, grade $3: 31 \%$ ), neutropenia (grade $1 / 2: 44 \%$, grade $3 / 4: 37 \%$ ), and thrombocytopenia (grade $1 / 2: 47 \%$, grade $3 / 4: 47 \%$ ). A total of $50 \%$ of patients required a blood transfusion for anaemia during the course of the study. No febrile neutropenia was reported. The most common non-haematologic $A E^{\prime} s$ were nausea/vomiting (grade 1/2: 91\%, grade 3: 3\%), fatigue (grades 1/2: $84 \%$ ), and constipation (grade 1/2: 34\%, grade 3: 3\%). Grades 3 and 4 AE's did not differ significantly between the breast and HGSOC cancer cohorts $(P=0.99)$. Supplementary Table 3 summarises worst grade $A E^{\prime}$ 's for different DL. Fig. 1 summarises the (A) haematological and (B) non-haematological $A E^{\prime} s$ experienced after each treatment cycle. The frequency of haematological $A E^{\prime}$ s increased with each treatment cycle, while the frequency of non-haematological $A E^{\prime}$ 's decreased with each cycle. Supplementary Tables 4 and 5 provide the details of dose modifications for olaparib and oral cyclophosphamide due to haematological AEs. 


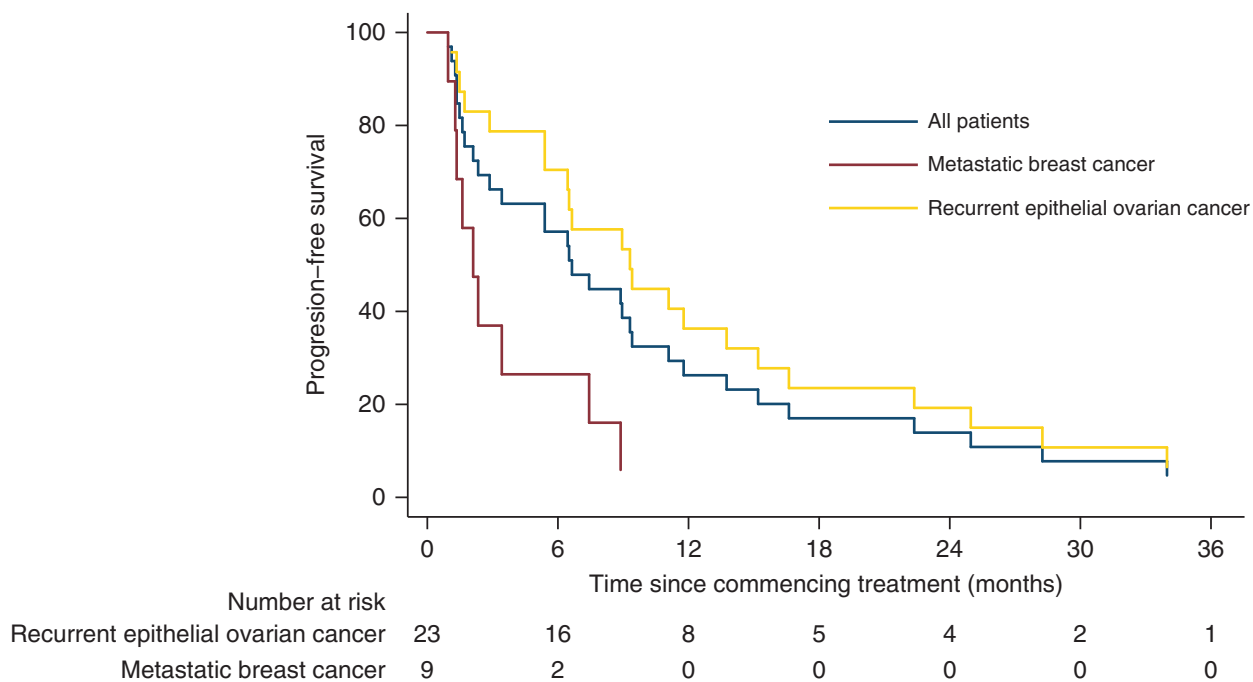

Fig. 3 Kaplan-Meier curves for progression-free survival for the overall population and the breast and ovarian cancer cohorts

Two (6\%) patients, treated at DL2, permanently discontinued treatment due to toxicity for anaemia (during cycle 5), and thrombocytopenia (during cycle 6), respectively. A total of 18 patients (56\%) completed eight cycles of protocol-defined therapy; 16 of these patients (50\%) continued beyond eight cycles outside protocol at the discretion of the study investigators. A total of 12 patients (38\%) terminated treatment before eight cycles of protocol-defined therapy due to PD.

Preliminary efficacy

In the ovarian cancer cohort $(N=23 ; \mathrm{g} B R C A m N=16), 21$ patients ( $g B R C A m N=14$ ) had evaluable disease by RECIST and 20 patients (gBRCAm $N=13$ ) were evaluable by GCIG CA125 criteria. Changes from baseline in tumour size and duration of response are shown in Fig. 2. ORR was 48\% (10/21) overall, and 64\% (9/14) in those with gBRCAm. SD6 was $81 \%(17 / 21)$ overall, $93 \%(13 / 14)$ in those with gBRCAm. GCIG CA125 response rate was 70\% (14/20) overall, and $92 \%(12 / 13)$ in those with gBRCAm. Median PFS was 9.4 months overall, and 11.8 months in the gBRCAm subset (Fig. 3). Supplementary Table 6 shows the relationship with response and platinum sensitivity in the ovarian cancer cohort.

In the breast cancer cohort $(N=9, \mathrm{~g} B R C A \mathrm{~m} N=6)$, all patients were evaluable by RECIST. No objective responses were observed, but SD6 was 56\% (5/9) overall, and 50\% (3/6) in those with gBRCAm. Median PFS was 2.1 months overall, and 1.3 months in the gBRCAm subset (Fig. 3).

Pharmacokinetic profiles

Pharmacokinetic (PK) data were available for five patients from the DL1 cohort. Before cyclophosphamide co-administration, the area under olaparib concentration curve (AUC) over time for olaparib monotherapy was 52.5 (95\% Cl: 44.5-61.9). There were no significant differences in the AUC with olaparib and cyclophosphamide co-administration (49.5, 95\% Cl: 42.1-58.1) (Supplementary Figure 1A). In the larger extension cohorts $(N=19)$, the AUCs with olaparib and cyclophosphamide co-administration were 55.8 (95\% Cl: $40.5-76.7)$ at day 7 of cycle 1 and 53.3 (95\% Cl: 39.2-72.5) at day 7 of cycle 2, respectively (Supplementary Figure 1B).

\section{DISCUSSION}

In this study, we have demonstrated that co-administration of olaparib tablets at the recommended dose of $300 \mathrm{mg}$ twice daily with metronomic oral cyclophosphamide $50 \mathrm{mg}$ on days $1-5$ weekly is safe and tolerable in patients with metastatic breast cancer and recurrent HGSOC. Our study utilised olaparib tablets rather than the capsule formulation (sixteen capsules daily) used in earlier trials; the dose of $300 \mathrm{mg}$ twice daily in tablet form (four tablets daily) has been established to be equivalent to $400 \mathrm{mg}$ twice daily in capsule form ${ }^{23}$ and is much more convenient for patients. Although no protocol-defined DLT events occurred during the first two cycles, no patient was recruited into the DL3 cohort because of concerns of haematological toxicity observed in patients in the later cycles. Despite the small number of patients and limited data availability, our pharmacokinetic studies suggested that olaparib exposure did not decrease with co-administration of metronomic oral cyclophosphamide. This study established the recommended RP2D dose of olaparib tablets as being $300 \mathrm{mg}$ twice daily, and cyclophosphamide tablets $50 \mathrm{mg}$ on days $1-5$ weekly.

Myelosuppression was the most significant toxicity associated with combination olaparib-cyclophosphamide therapy. In our study, we observed high rates of grade 3 anaemia (31\%), grade $3 /$ 4 neutropenia (37\%), grade $3 / 4$ lymphopenia (75\%) and grade $3 / 4$ thrombocytopenia (47\%). Although $50 \%$ of patients required a blood transfusion for anaemia during the course of the study, there was no reported febrile neutropenia. In contrast, previous olaparib monotherapy studies ${ }^{1-7}$ reported rates of grade $3 / 4$ anaemia ranging from 3 to $17 \%$, neutropenia of $9 \%$, lymphopenia of 8 to $12 \%$ and no grade $3 / 4$ for thrombocytopenia. Oral cyclophosphamide monotherapy is associated with low rates of myelosuppression with one recent study reporting $8 \%$ of grade 3 lymphopenia as the most significant haematological toxicity. ${ }^{20}$ In a randomised Phase 2 trial, veliparib $(60 \mathrm{mg}$ daily) and oral cyclophosphamide (50 mg daily) was associated with rates of grade 3 anaemia of $5 \%$, neutropenia of $5 \%$, lymphopenia of $35 \%$, and thrombocytopenia of $5 \% .{ }^{20}$ Importantly, that study investigated veliparib at a dose that is significantly lower than used in current trials. For example, veliparib doses have ranged from 250 to $400 \mathrm{mg}$ twice daily when used either as monotherapy or in combination with platinum-based chemotherapy. It is therefore unclear whether the dose of veliparib $60 \mathrm{mg}$ daily has any therapeutic impact on any $\mathrm{gBRCAm}$ and other homologous recombination-deficient cancers.

Non-haematologic toxicities, namely nausea/vomiting, fatigue, and constipation were generally of low grades. Although these low-grade toxicities might have a major impact in patients undergoing continuous treatment administered chronically, our trial suggests that the frequency and severity of these toxicities decrease over time (Fig. 1b). Importantly, the two patients (6\%) 
who permanently discontinued the study treatment due to AEs were haematological rather than non-haematological in nature.

This study has also demonstrated promising preliminary evidence of anti-tumour activity with a combination of olaparib and metronomic cyclophosphamide in the HGSOC cohort. No significant activity was demonstrated in the breast cancer cohort, but the small number of patients treated precludes any conclusion regarding efficacy in this group.

This study has several strengths. We adopted the strategy of maintaining the recommended monotherapy dose of olaparib tablets while modifying the scheduling of cyclophosphamide. In keeping with data from multiple studies, the efficacy of olaparib monotherapy in homologous recombination-deficient cancers has already been well demonstrated, and hence our treatment strategy avoided any compromise in olaparib dosing. Unlike previous studies that utilised platinum and other chemotherapeutic agents, which were limited due to significant myelotoxicity, our choice of oral cyclophosphamide as the DNA-damaging agent has revealed acceptable tolerability despite higher rates of anaemia, neutropenia, and lymphopenia than reported with olaparib monotherapy. However, there are also several limitations. It is not possible to draw definitive conclusions regarding efficacy of olaparib and metronomic oral cyclophosphamide combination in this phase 1 trial. We do not fully understand whether the apparent synergistic anti-tumour activity with this combination is mediated through enhanced PARP trapping with olaparib.

In conclusion, olaparib and metronomic oral cyclophosphamide is relatively well-tolerated, with an acceptable safety profile. Owing to the encouraging preliminary evidence of efficacy, a multi-centre, randomised Phase 2 study in patients with advanced HGSOC with and without gBRCAm will commence shortly.

\section{ACKNOWLEDGEMENTS}

We acknowledge the editorial support provided by Dr Sherilyn Goldstone, PhD (NHMRC Clinical Trials Centre). We thank the participating patients and their families.

\section{ADDITIONAL INFORMATION}

Supplementary information is available for this paper at https://doi.org/10.1038/ s41416-018-0349-6.

Competing interests: The authors declare no competing interests.

Note: This work is published under the standard license to publish agreement. After 12 months the work will become freely available and the license terms will switch to a Creative Commons Attribution 4.0 International licence (CC BY 4.0).

\section{REFERENCES}

1. Audeh, M. W. et al. Oral poly(ADP-ribose) polymerase inhibitor olaparib in patients with BRCA1 or BRCA2 mutations and recurrent ovarian cancer: a proofof-concept trial. Lancet 376, 245-251 (2010).

2. Kaufman, B. et al. Olaparib monotherapy in patients with advanced cancer and a germline BRCA1/2 mutation. J. Clin. Oncol. 33, 244-250 (2015).
3. Gelmon, K. A. et al. Olaparib in patients with recurrent high-grade serous or poorly differentiated ovarian carcinoma or triple-negative breast cancer: a phase 2, multicentre, open-label, non-randomised study. Lancet Oncol. 12, 852-861 (2011).

4. Fong, P. C. et al. Inhibition of poly(ADP-ribose) polymerase in tumors from BRCA mutation carriers. New Engl. J. Med. 361, 123-134 (2009).

5. Robson, M. et al. Olaparib for metastatic breast cancer in patients with a germline BRCA mutation. New Engl. J. Med. 377, 523-533 (2017).

6. Fong, P. C. et al. Poly(ADP)-ribose polymerase inhibition: frequent durable responses in BRCA carrier ovarian cancer correlating with platinum-free interval. J. Clin. Oncol. 28, 2512-2519 (2010).

7. Kaye, S. B. et al. Phase II, open-label, randomized, multicenter study comparing the efficacy and safety of olaparib, a Poly (ADP-ribose) polymerase inhibitor, and pegylated liposomal doxorubicin in patients with BRCA1 or BRCA2 mutations and recurrent ovarian cancer. J. Clin. Oncol. 30, 372-379 (2012).

8. Evers, B. et al. Selective inhibition of BRCA2-deficient mammary tumor cell growth by AZD2281 and cisplatin. Clin. Cancer Res. 14, 3916-3925 (2008).

9. Rottenberg, S. et al. High sensitivity of BRCA1-deficient mammary tumors to the PARP inhibitor AZD2281 alone and in combination with platinum drugs. Proc. Natl Acad. Sci. USA 105, 17079-17084 (2008).

10. Oza, A. M. et al. Olaparib combined with chemotherapy for recurrent platinumsensitive ovarian cancer: a randomised phase 2 trial. Lancet Oncol. 16, 87-97 (2015).

11. Balmaña, J. et al. Phase I trial of olaparib in combination with cisplatin for the treatment of patients with advanced breast, ovarian and other solid tumors. Ann. Oncol. 25, 1656-1663 (2014).

12. Lee, J.-M. et al. Phase $\mathrm{I} / \mathrm{lb}$ study of olaparib and carboplatin in BRCA1 or BRCA2 mutation-associated breast or ovarian cancer with biomarker analyses. J. Natl Cancer Inst. 106, dju089 (2014).

13. Dent, R. A. et al. Phase I trial of the oral PARP inhibitor olaparib in combination with paclitaxel for first- or second-line treatment of patients with metastatic triple-negative breast cancer. Breast Cancer Res. 15, R88-R88 (2013).

14. Colleoni, M. et al. Metronomic low-dose oral cyclophosphamide and methotrexate plus or minus thalidomide in metastatic breast cancer: antitumor activity and biological effects. Ann. Oncol. 17, 232-238 (2006).

15. Colleoni, M. et al. Low-dose oral methotrexate and cyclophosphamide in metastatic breast cancer: antitumor activity and correlation with vascular endothelial growth factor levels. Ann. Oncol. 13, 73-80 (2002).

16. Garcia, A. A. et al. Phase II clinical trial of bevacizumab and low-dose metronomic oral cyclophosphamide in recurrent ovarian cancer: a trial of the California, Chicago, and Princess Margaret Hospital Phase II Consortia. J. Clin. Oncol. 26, 76-82 (2008)

17. Norris, R. E., Adamson, P. C., Nguyen, V. T. \& Fox, E. Preclinical evaluation of the PARP inhibitor, olaparib, in combination with cytotoxic chemotherapy in pediatric solid tumors. Pediatr. Blood. Cancer 61, 145-150 (2014).

18. Hopkins, T. A. et al. Mechanistic dissection of PARP1 trapping and the impact on in vivo tolerability and efficacy of PARP inhibitors. Mol. Cancer Res. 13, 1465-1477 (2015).

19. Murai, J. et al. Trapping of PARP1 and PARP2 by clinical PARP inhibitors. Cancer Res. 72, 5588-5599 (2012).

20. Kummar, S. et al. Randomized trial of oral cyclophosphamide and veliparib in high-grade serous ovarian, primary peritoneal, or fallopian tube cancers, or BRCAmutant ovarian cancer. Clin. Cancer Res. 21, 1574-1582 (2015).

21. Vergote, I. et al. Re: New guidelines to evaluate the response to treatment in solid tumors [Ovarian Cancer]. J. Natl. Cancer Institute 92, 1534-1535 (2000).

22. Roth, J. et al. A sensitive and robust ultra HPLC assay with tandem mass spectrometric detection for the quantitation of the PARP inhibitor olaparib (AZD2281) in human plasma for pharmacokinetic application. Chromatography 1, 82 (2014).

23. Mateo, J. et al. An adaptive study to determine the optimal dose of the tablet formulation of the PARP inhibitor olaparib. Target. Oncol. 11, 401-415 (2016). 